\title{
THE ROLE OF COSMIC RAYS AND ALFVÉN WAVES IN THE STRUCTURE OF THE GALACTIC HALO
}

\author{
M. K. DOUGHERTY* and J. F. MCKENZIE ${ }^{\dagger}$ \\ *Max-Planck-Institut für Kernphysik, Postfach 103980, \\ D-6900 Heidelberg 1, FRG \\ ${ }^{\dagger}$ Max-Planck-Institut für Aeronomie, Postfach 20, \\ D-3411 Katlenburg-Lindau 3, FRG
}

\begin{abstract}
The effect that cosmic rays and the Alfven waves generated by them have on the structure of the plasma distribution perpendicular to the galactic disc in a hydrostatic model is examined. It is shown that the plasma distribution exhibits two length scales, essentially because the cosmic rays and Alfvén waves lift the gas up and stretch it out beyond the galactic plane. The predicted gas density far from the galactic plane indicates that models involving hydrostatic equilibrium should be replaced by those allowing for a galactic wind.
\end{abstract}

\section{Introduction}

Since the energy densities of the three components of the interstellar medium, that of the plasma, magnetic field and cosmic rays are of the same order (Ghosh and Ptuskin, 1983, hereafter referred to as $G P$ ) the coupling between them must be taken into account in a self-consistent fashion. In this paper we consider a hydrostatic model of the halo and show that the cosmic rays and Alfvén waves can play a dominant role in the behaviour of the plasma distribution perpendicular to the disc.

\section{The Theory}

A simple one-dimensional model is considered where the basic assumption is that of hydrostatic equilibrium perpendicular to the plane of the disc. The potential which gives the gravitational acceleration is the same as in $G P$ and consists of a concentrated nucleus combined with a more distributed stellar population. The cosmic rays are treated hydrodynamically in the sense that they are described by a pressure and adiabatic index and an effective diffusion constant $\kappa$ (McKenzie and Völk, 1982), and the cosmic ray transport equation incorporates Alfvénic effects as well as those of convection and diffusion. The cosmic ray energy flux consists of the sum of the cosmic ray enthalpy flux and diffusive energy flux and its divergence is balanced by the rate at which cosmic rays lose energy in generating Alfvén waves through recoil upon scattering. The $z$-component of the galactic magnetic field is assumed to be constant. The system is completed by the wave energy exchange equation which describes how the wave pressure is generated by the cosmic ray pressure gradient. The sum of these equations gives a total energy flux conservation law. 


\section{The Results}

Analytic results can be obtained in two limits. In the strong scattering mode where it is assumed that the diffusive flux $\ll$ the cosmic ray enthalpy flux, the model predicts that the plasma distribution will exhibit two length scales. Close to the galactic plane there is a rapid fall-off in density whose scale height is determined by the gas (and magnetic) pressure, this is followed by a much slower decrease determined by the cosmic ray and Alfvén wave pressure. In physical terms the existence of two length scales can be attributed to the fact that the cosmic rays and Alfven waves that are excited by them lift the gas up and stretch it far out beyond the galactic plane; this feature is consistent with galactic models with a halo (Ginzburg and Ptuskin, 1976). Asymptotically the plasma density approaches a small non-zero value which is much larger than typical background intergalactic density values, with the implication that a galactic wind should probably occur. In the limit of weak scattering the cosmic ray energy flux is neglected and the work of $G P$ is extended to include the effects of Alfven waves. Our model suffers from the same defect as the GP model in that sufficiently far from the disc the plasma density and wave pressure increase and the cosmic ray pressure decreases and becomes negative. This arises since it is inconsistent to assume that the diffusive energy flux remains constant because the wave energy flux grows at the expense of the diffusive energy flux so that the system will drive itself into the strong scattering mode. A simple perturbation analysis, carried out by Dougherty, 1988, which examines the effects of $\kappa$, the effective diffusion constant, on the system shows that diffusion decreases the effect of the cosmic rays on the plasma density because they eventually become decoupled.

\section{Conclusions}

The influence of cosmic rays and Alfven waves on the structure of the plasma perpendicular to the galactic disc was examined. The strong scattering limit revealed that the plasma distribution falls off according to two distinct length scales. This model indicates that a totally self-consistent solution will entail a galactic wind. To some extent this analysis has been overtaken by models of the galactic wind (McKenzie et.al, 1987 and Breitschwerdt et.al, 1987).

\section{References}

Breitschwerdt, D., Völk, H. J. and McKenzie, J. F. (1987), 'Cosmic Ray and Wave Driven Galactic Wind Solutions', Proceedings of the 20'th Int. Cosmic Ray Conf. (Moscow) 2, 115-118.

Dougherty, M. K. (1988), 'Wave Particle Interactions in Anisotropic and Dispersive Media', Ph.D Thesis, University of Natal, Durban, South Africa.

Ghosh, A. and Ptuskin, V. S. (1983), 'The Role of Cosmic Rays in Hydrostatic Equilibrium of the Galactic Halo', Astrophysics and Space Science 92, 37-44.

Ginzburg, V. L. and Ptuskin, V. S. (1976), 'On the origin of cosmic rays : Some problems in high-energy astrophysics', Reviews of Modern Physics 48, 161-189.

McKenzie, J. F., Breitschwerdt, D. and Völk, H. J. (1987), 'Flux Tube Formulation of Galactic Wind Equations Including Cosmic Rays and Waves', Proceedings of the 20'th Int. Cosmic Ray Conf. (Moscow) 2, 119-122.

McKenzie, J. F. and Völk, H. J. (1982), 'Non-linear Theory of Cosmic Ray Shocks Including Self-generated Alfvén Waves', Astronomy and Astrophysics 116, 191-200. 